\title{
TRANSITIVITY OF THE AUTOMORPHISMS OF CERTAIN GEOMETRIC STRUCTURES
}

\author{
BY \\ WILLIAM M. BOOTHBY $\left({ }^{1}\right)$
}

1. Introduction. We suppose $\Gamma$ to be an infinite Lie transformation group acting on $R^{n}$, or somewhat more precisely, a pseudogroup of $C^{\infty}$ diffeomorphisms from open subsets of $R^{n}$ to open subsets of $R^{n}$ satisfying a suitable system of differential equations. We will not give exact definitions since we are concerned only with some very familiar examples; for more careful definitions, see Chern [1] and Kobayashi and Nomizu [3]. The examples we discuss are the following: (1) $\Gamma$ is the collection of all holomorphic diffeomorphisms of open subsets of $R^{2 m}$, identified with $C^{m}$, onto such open sets; (2) $\Gamma$ consists of diffeomorphisms of open sets of $R^{n}$ which leave invariant the volume element $d x_{1} \wedge \cdots \wedge d x_{n}$, i.e., diffeomorphisms whose Jacobian has the value +1 ; (3) $\Gamma$ consists of those diffeomorphisms leaving invariant the closed quadratic exterior form $\sum_{j=1}^{m} d x_{j} \wedge d x_{j+m}, n=2 m$; and finally, (4) $\Gamma$ consists of those diffeomorphisms on $R^{n}, n=2 m+1$, which leave invariant to within a nonvanishing scalar multiple the exterior 1 -form $d x_{0}+$ $\sum_{j=1}^{m} x_{j} \wedge d x_{m+j}$. It is well known that the collection of such diffeomorphisms on open subsets of $R^{n}$ determine in each case above a pseudogroup which is locally transitive, that is, each point $x$ has a neighborhood $V$ such that if $y \in V$, then there is an element $\gamma$ of $\Gamma$ taking $x$ to $y$. This is obvious in the first three cases, since translations of $R^{n}$ are in each of the three pseudogroups; it is a consequence of arguments below in the fourth case.

Any pseudogroup $\Gamma$ determines a corresponding $\Gamma$-structure on a manifold $\left({ }^{2}\right)$ provided that it is possible to cover the manifold with coordinate neighborhoods in such a fashion that the change of coordinates is given by elements of the pseudogroup. In the case of our examples the manifolds so determined are (1) complex analytic manifolds; (2) manifolds with a volume element $\Theta$; (3) manifolds with a closed exterior quadratic form $\Omega$ of maximum rank, i.e. with $d \Omega=0$ and $\Omega^{m} \neq 0$, $2 m=n=\operatorname{dim} M$ (this is called a symplectic structure); and (4) manifolds on which there is defined a 1 -form $\omega$ with $\omega \wedge d \omega^{m} \neq 0,2 m+1=n=\operatorname{dim} M$ (these manifolds

Presented to the Society, August 31, 1967 under the title Transitivity of the automorphism groups of some geometric structures on manifolds; received by the editors December 16, 1967.

$\left.{ }^{1}\right)$ The author is grateful for the support of Washington University during a sabbatical leave for the academic year 1965-1966 during which these results were obtained, and for the support of the National Science Foundation and the American-Swiss Foundation for Scientific Exchange during this period.

( $\left.{ }^{2}\right)$ Manifolds are assumed connected and $C^{\infty}$ but not necessarily compact; functions and maps are $C^{\infty}$. 
are sometimes called contact manifolds). Any diffeomorphism $\Phi: M \rightarrow M$ which preserves the structure, i.e. is complex analytic, volume preserving, etc. will be called an automorphism of $M$; the automorphisms of a given structure will, of course, be a subgroup of the group of diffeomorphisms. In this note we consider the question of whether the group of automorphisms in our examples acts transitively on $M$. The first case was included in order to show that the answer is sometimes negative: if $M$ is a closed Riemann surface of genus $>1$, then the group of complex analytic diffeomorphisms is known to be finite; thus it cannot be transitive. However, in what follows we show that in the other three cases the automorphism group is $k$-fold transitive for any natural number $k$.

ADDENDUM. The author is indebted to the referee for pointing out that related results for symplectic and contact manifolds were obtained in the compact case by Y. Hatakeyama [7].

2. General considerations. Let $M$ be a manifold with a $\Gamma$-structure as defined above and let $p_{1}, \ldots, p_{k}$ and $q_{1}, \ldots, q_{k}$ be any two collections of distinct points of $M$. We say that $M$ is $k$-fold transitive if there is an automorphism $\Phi: M \rightarrow M$ such that $\Phi\left(p_{j}\right)=q_{j}, j=1, \ldots, k$. By definition, $\Phi$ is a diffeomorphism preserving the $\Gamma$-structure. Automorphisms are said to be isotopic in this note if they are $\Gamma$-isotopic, that is, if there is a $C^{\infty}$ homotopy $H: M \times I \rightarrow M$ between them such that for each $0 \leqq t \leqq 1$ the map $x \rightarrow H(x, t)$ is an automorphism of the structure, and $H(x, 0), H(x, 1)$ correspond to the given automorphisms. The following proposition is well known and is easy to prove in the case of diffeomorphisms; the proof is the same here, see Milnor [5].

Proposition. On a $\Gamma$-manifold $\Gamma$-isotopy is an equivalence relation on the automorphisms.

It is only the transitivity of the equivalence relation that presents any problem. Suppose that $\Phi_{0}$ and $\Phi_{1}$ are isotopic by $H(x, t)$ and $\Phi_{1}$ and $\Phi_{2}$ are isotopic by $G(x, t)$ respectively. Let $f: I \rightarrow I$ be a $C^{\infty}$ function on the unit interval such that for $0 \leqq t \leqq 1 / 4$, we have $f(t) \equiv 0$, for $3 / 4 \leqq t \leqq 1$ we have $f(t) \equiv 1$, and for all other values $f$ is monotone increasing. Then $H(x, f(t))$ and $G(x, f(t))$ are $\Gamma$ isotopies of the same maps $\Phi$, as before; but it is seen at once that they can be composed to give a $C^{\infty}$ isotopy of $\Phi_{0}$ to $\Phi_{2}$. It is important to note that if a point $x$ is left fixed for all $t$ by both of the original isotopies, then the same will be true for the smooth isotopy of $\Phi_{0}$ to $\Phi_{2}$, which we thus define.

To simplify certain statements we introduce the following definition: we shall say that the automorphisms of a $\Gamma$-manifold $M$ are strongly locally transitice on $M$ if for each $p \in M$ and neighborhood $U$ of $p$ there are neighborhoods $V$ and $W$ of $p$ with $\bar{V} \subset W$ and $\bar{W} \subset U, \bar{W}$ compact, and for any $q \in V$ there is a $\Gamma$-isotopy $\Phi_{t}$ of the identity map $\Phi_{0}$ to $\Phi_{1}$ such that (i) $\Phi_{1}(p)=q$ and (ii) for all $t$, $\Phi_{t}$ leaves fixed every point outside $\bar{W}$. Note that it is really a local property since if it is known to hold for $U$, then the isotopy $\Phi_{t}$ of $U$ can be extended to all of $M$ by defining it to be the 
identity outside $U$. Since it is already the identity outside $\bar{W}$ on $U$, this is obviously a $C^{\infty}$ extension of the isotopy. Our treatment of the three cases is not completely unified, but the same ideas are used in each and are embodied in the following lemma, which, together with the preceding remark, will enable us to reduce our problem to one to be solved in a coordinate neighborhood.

LEMMA. Suppose that the $\Gamma$-structure on $M$ is strongly locally transitive. Then for any two sets $p_{1}, \ldots, p_{k}$ and $q_{1}, \ldots, q_{k}$ of distinct points of $M$ and any natural number $k$, there is an automorphism $\Phi_{1}$ of $M$ with $\Phi_{1}\left(p_{j}\right)=q_{j}, j=1, \ldots, k . \Phi_{1}$ is $\Gamma$-isotopic to the identity $\Phi_{0}$ by an isotopy $\Phi_{t}, 0 \leqq t \leqq 1$ leaving every point of $M$ fixed except for a set of arbitrarily small volume $\left({ }^{3}\right)$.

Proof. Let $C_{1}$ be a path from $p_{1}$ to $q_{1}$ which does not contain any of the points $p_{2}, \ldots, p_{k}$ or $q_{2}, \ldots, q_{k}$ and let $\varepsilon>0$ be so chosen that the $\varepsilon$-neighborhood $\left.{ }^{3}\right) U_{1}$ of $C_{1}$ has volume less that $v_{0} / k, v_{0}$ being any preassigned positive number, and also so that $U_{1}$ does not contain the points excluded from $C_{1}$ above. For each $x \in C_{1}$ we choose $\delta_{x}^{\prime}$ and $\delta_{x}^{\prime \prime}$ with $0<\delta_{x}^{\prime}<\delta_{x}^{\prime \prime}<\varepsilon$ so that the $\delta^{\prime}$ and $\delta^{\prime \prime}$ neighborhoods of $x$ satisfy the conditions of $V, W$ in the definition of strong local transitivity. Since $C_{1}$ is compact, there is a finite collection of points $p_{1}=a_{1}, a_{2}, \ldots, a_{r}=q_{1}$ along $C_{1}$ whose corresponding $\delta^{\prime}$-neighborhoods cover $C_{1}$. By using the assumed existence of the "local" isotopies of $M$ which move only points in these neighborhoods, and the transitivity of isotopy, we may find a $\Gamma$-isotopy which is the identity for $t=0$ and takes $p_{1}$ to $q_{1}$ for $t=1$ and is, for all $t$, the identity on all points outside the union of the closures of the $W$-neighborhoods of $a_{1}, \ldots, a_{r}$. This union is a compact set which lies interior to $U_{1}$. Let it be denoted by $F_{1}$ and the isotopy by $H_{1}(x, t)$. Since $M-F_{1}$ is a connected $\Gamma$-manifold containing the points $p_{2}, \ldots, p_{k}$ and $q_{2}, \ldots, q_{k}$, we may apply the same argument and obtain an isotopy $H_{2}(x, t)$ connecting the identity to an automorphism taking $p_{2}$ to $q_{2}$ and carried by a compact set $F_{2}$ of volume less than $v_{0} / k$. Proceeding in this fashion for $k$ steps and then defining an isotopy $H(x, t)=H_{j}(x, t)$ for $x \in U_{j}$ and the identity outside the compact set $F_{1} \cup \cdots \cup F_{k}$, we obtain the desired automorphism $\Phi_{1}=H(x, 1)$ and its isotopy to the identity, $H(x, 0)$. Note that this isotopy is the identity map on $M$ except for the set $F_{1} \cup \cdots \cup F_{k}$ whose volume is less than $v_{0}$. By use of this lemma, or very similar arguments, we may reduce the proofs of $k$-fold transitivity in the separate cases to essentially local arguments which we can restrict to coordinate neighborhoods. This is done in the next two sections.

3. Manifolds with symplectic structure or a volume element. In the case of symplectic structure given by a closed quadratic form $\Omega$ of maximum rank it is well known that in a neighborhood $U$ of any point $p_{0}$ it is possible to introduce local coordinates relative to which $\Omega=\sum_{j=1}^{m} d x_{j} \wedge d x_{j+m}$. We may suppose that the

$\left({ }^{3}\right)$ We choose arbitrarily a Riemannian metric on $M$. In particular, this determines a volume element. 
image of $U$ in $R_{n}, n=2 m$, is an $\varepsilon$-ball $U^{\prime}$ around the point $x_{0}$ corresponding to $p_{0}$. We shall use this neighborhood to prove strong local transitivity with $W^{\prime}$ and $V^{\prime}$ taken to be $\varepsilon / 2$ and $\varepsilon / 3$-balls respectively. Suppose $y \in V^{\prime}$, let $X^{\prime}$ be the vector field of all vectors parallel to $x_{0} y^{\prime}$; it will generate a one parameter group $\Phi_{t}^{\prime}$ of translations of $R^{n}$ all of which leave the form $\sum_{j=1}^{m} d x_{j} \wedge d x_{j+m}$ invariant and such that $\Phi_{0}^{\prime}$ is the identity and $\Phi_{1}^{\prime}$ is the translation taking $x_{0}$ to $y$. For $0 \leqq t \leqq 1, \Phi_{t}^{\prime}$ is an isotopy between these two automorphisms of the symplectic form on $R^{n}$. Now it is well known that a vector field $X$ on a symplectic manifold $M$ is the infinitesimal generator of a 1-parameter group of automorphisms if and only if the 1-form $i(X) \Omega$ is closed. Since the condition that $X$ generate a group leaving $\Omega$ invariant is exactly that the Lie derivative $L_{X} \Omega=0$, the statement just made follows from the identity (see [3])

$$
L_{X}=i(X) d+d i(X),
$$

connecting the Lie derivative relative to the vector field $X$ and the skew derivations $d$ and $i(X)$ on the ring of differential forms. For in fact, $d \Omega$ being zero everywhere gives $L_{X} \Omega=d i(X) \Omega$, which proves the statement. This characterization of the infinitesimal automorphisms and others used below may be found in Libermann [4].

Now let $f$ be a $C^{\infty}$ function on $U^{\prime}$ such that $f(x) \equiv 1$ on $V^{\prime}$ and $\equiv 0$ outside $\bar{W}^{\prime}$ and is $\geqq 0$ for all $x$. Using the Poincare Lemma, we know that there is a function $g(x)$ on $U^{\prime}$ such that $d g=i\left(X^{\prime}\right) \Omega$. Let $\sigma$ denote the form $d(f g)$ on $U^{\prime}$ and $X$ the vector field defined by $i(X) \Omega=\sigma$ on $U^{\prime}$. This vector field is exactly the same as $X^{\prime}$ on $V^{\prime}$ and it is identically zero outside $\bar{W}^{\prime}$, so that if we denote by $V, W$ respectively the images of $V^{\prime}, W^{\prime}$ in the neighborhood $U$ on $M$ and by $X$ the image of this vector field, then $X$ may be extended to all of $M$ by setting it equal to zero outside $\bar{W}$. As such, it is a $C^{\infty}$ vector field on $M$ which generates a 1-parameter group $\Phi_{t}$ of automorphisms of $M$. For all $t, \Phi_{t}$ is the identity outside $\bar{W}$ and for $t=1$ it is an automorphism which carries $p_{0}$ to the image $q$ of $y$. $\Phi_{t}$ for $0 \leqq t \leqq 1$ gives an isotopy of this automorphism with the identity. This establishes the strong local transitivity of the group of automorphisms of $M$.

In the case of a manifold with a volume element we are able to obtain a considerably stronger result. Let $C$ be a differentiably imbedded image in $M$ of the unit interval $I$ or of the circle $S^{1}$. Using a metric on $M$, for small values of $\varepsilon>0$ there exists a tubular neighborhood $U$ of $C$ formed by geodesic segments of length $<\varepsilon$ whose initial points are on $C$ and whose initial direction is orthogonal to $C$. This neighborhood may be mapped diffeomorphically onto $I \times B^{n-1}$ whenever $C$ is the image of $I$, and $S^{1} \times B^{n-1}$ when $C$ is the image of $S^{1}$ and $n>1, B^{n-1}$ denoting the open disk $\{x \mid\|x\|<1\}$ in $R^{n-1}$. The existence of this diffeomorphism, which we denote $\phi$, depends on the contractibility of $C$ in the first instance and on the parallelizability of the normal bundle to $S^{1} \subset M$, an orientable manifold, in the second case; the fibre, i.e. points on geodesics issuing from a single point of $C$ maps onto points along radial lines in $t \times B^{n-1}$ and $C$ itself onto the set $I \times(0, \ldots, 0)$ 
where $x_{1}, \ldots, x_{n-1}$ denote the coordinates on the disk. Interior to the tubular neighborhood $U$ we may define open sets $V$ and $W$ with $\bar{V} \subset W$ and $\bar{W} \subset U$ as follows. In the case of $S^{1}$ we simply take smaller tubular neighborhoods of radii $\varepsilon / 3$ and $\varepsilon / 2$ respectively. In the case of the arc we suppose $a, b$ to be the endpoints and $p, q$ to be interior points in the order $a, p, q, b$. Choose points $a^{\prime}, p^{\prime}$ between $a$ and $p$ and $q^{\prime}, b^{\prime}$ between $q$ and $b$ and let $W$ be the portion of a tubular neighborhood of radius $\varepsilon / 2$ cut off by the fibres through $a^{\prime}, b^{\prime}$, and $V$ the portion of a tubular neighborhood of radius $\varepsilon / 3$ cut off by the fibres through $p^{\prime}, q^{\prime}$ respectively. We let $t, 0 \leqq t \leqq 1$, or $t$ mod $2 \pi$ respectively, denote the parameter along $C$ in the cases of the segment $I$ and the circle $S^{1}$. Then $\Theta=f d t \wedge d x_{1} \wedge \cdots \wedge d x_{n-1}$, where $f$ is a positive function of $\left(t, x_{1}, \ldots, x_{n-1}\right)$, periodic in $t$ in the case where $C$ is closed. We will further denote by $I^{\prime}$ the portion of the unit interval which corresponds to the part $C^{\prime}$ of $C$ between $p^{\prime}$ and $q^{\prime}$. We will define a vector field on $M$ tangent to $C$ which is of unit length on $C^{\prime}$ when $C$ is an arc, on all of $C$ when $C$ is closed, and which vanishes outside the compact set $\bar{W}$. Moreover we arrange that the 1-parameter group which it generates leaves the volume element invariant. This is guaranteed if $L_{X} \Theta=0$, or using a similar argument to the symplectic case, if and only if the form $i(X) \Theta$ is closed: any closed $n-1$ form determines such an $X$, and conversely. On $I \times B^{n-1}$ or $S^{1} \times B^{n-1}$ we let $X^{\prime}=(1 / f) \partial / \partial t$, then it is immediate that $i\left(X^{\prime}\right) f d t \wedge d x_{1} \wedge \cdots \wedge d x_{n-1}$ $=d x_{1} \wedge \cdots \wedge d x_{n-1}$ is closed. However, because these spaces are contractible to a point, or to $S^{1}$ respectively, any closed form of degree greater than 0 , or 1 respectively, is exact. Let $\sigma$ be an $n-2$ form such that $d \sigma=i\left(X^{\prime}\right) f d t \wedge d x_{1} \cdots \wedge d x_{n-1}$ and choose a $C^{\infty}$ function $g$, which is identically 1 on $V$ and identically 0 outside $\bar{W}$. Then $g \sigma \equiv \sigma$ on $V$ and thus the vector field $X$ defined by $i(X) f d t \wedge d x_{1} \wedge \cdots \wedge d x_{n-1}$ $=d(g \sigma)$ agrees with $X^{\prime}$ on $V$ and generates a 1-parameter group of automorphisms $\Phi_{t}$, which is the identity outside $\bar{W}$ and which moves points of $C^{\prime} \cap V$ along itself but has $C$ as a closed orbit in the case where $C$ is a closed curve, or takes $p$ to $q$ when $t=1$ in the case of an arc. As before, the group $\Phi_{t}$ defines an isotopy between the identity, $\Phi_{0}$, and the automorphisms $\Phi_{1}$ in the former case. Clearly, by choosing $\varepsilon$ small enough, we may make the volume of $\bar{W}$ as small as we wish.

We could use the above results on volume elements to prove strong local transitivity, but it is simpler to proceed more directly. Suppose $p_{1}, \ldots, p_{k}$ and $q_{1}, \ldots, q_{k}$ are two sets of distinct points of $M$, a manifold with volume element $\Theta$. We may choose paths $C_{1}, \ldots, C_{k}$, each of which is a differentiably imbedded image of $I$ with $p_{j}$ and $q_{j}$ as interior points of $C_{j}$ and so chosen that no two of these arcs have a common point. Then taking $\varepsilon>0$ small enough we may find for each a tubular neighborhood $U_{j}$ such that these neighborhoods are pairwise disjoint and their total volume less than a preassigned number. Then defining $X$ separately on each of them as above and taking it to be zero outside these neighborhoods, we generate a 1-parameter group $\Phi_{t}$ such that $\Phi_{t}$ takes $p_{j}$ to $q_{j}$ for $j=1, \ldots, k$ and such that for $0 \leqq t \leqq 1, \Phi_{t}$ defines an isotopy of $\Phi_{1}$ and the identity $\Phi_{0}$. By its method of construction, $\Phi_{t}$ leaves invariant the volume element for all values of $t$. 
THEOREM A. If $M$ is a symplectic manifold or a manifold with a volume element and $p_{1}, \ldots, p_{k}$ and $q_{1}, \ldots, q_{k}$ are two sets of distinct points of $M$, then there is a symplectic or volume preserving transformation $\Phi\left(p_{j}\right)=q_{j}, j=1, \ldots, k$, which is isotopic to the identity by an isotopy which preserves the structure and leaves fixed every point of $M$ outside a compact set of arbitrarily small volume.

We remark that if the arguments just used are applied to the case of closed curves we have the following:

If $M$ is a manifold with volume element and its dimension is $\geqq 2$, and if, moreover, $C_{1}, \ldots, C_{k}$ is an arbitrary collection of disjoint closed curves on $M$, each a differentiably imbedded image of $S^{1}$, then there is a 1-parameter group of volume preserving transformations on $M$ with these curves as orbits.

4. Manifolds with contact structure. We recall that this structure on $M$ is equivalent to a 1 -form $\omega$ defined to within a nonvanishing scalar multiple and satisfying $\omega \wedge d \omega^{n} \neq 0$. An integral curve of $\omega$ on $M$ is a differentiable or piecewise differentiable curve on whose tangent vectors $\omega=0$, or equivalently a curve such that the restriction to the curve of $\omega$ vanishes. In order to prove strong local transitivity we will need the following:

Lemma. Let $C$ be a differentiably imbedded image of the closed interval I which is an integral curve of a contact form $\omega$ on a manifold $M$ of dimension $n=2 m-1$. If $p, q$ are interior points of $C$, then there is a 1-parameter group of transformations $\Phi_{t}$ acting on $M$ and leaving invariant the form $\omega$ to within a scalar multiple and such that $\Phi_{t}$ has a portion of $C$ containing $p$ and $q$ as orbit but leaves fixed all points outside an $\varepsilon$-neighborhood of this portion. Hence there is a contact transformation $\Phi_{1}$ on $M$ which takes $p$ to $q$ and is isotopic by $\Phi_{t}, 0 \leqq t \leqq 1$, to the identity $\Phi_{0}$.

Proof. Let $a, b$ denote the endpoints of $C$ and taking a sufficiently small $\varepsilon>0$ we let $U$ denote an $\varepsilon$-tubular neighborhood of $C$. Let $C^{\prime}$ denote the portion between $p$ and $q$ and choose $a^{\prime}, b^{\prime}, p^{\prime}, q^{\prime}$ and neighborhoods $V, W$, with $\bar{V} \subset W$ and $\bar{W} \subset U$ exactly as in the preceding section where we used tubular neighborhoods of an arc. We will suppose $C$ is parametrized by $z, 0 \leqq z \leqq 3$ with $p$ corresponding to $z=1$ and $q$ to $z=2$. Along $C$ we take a $C^{\infty}$ field of tangent vectors $Y_{z}$ which is of unit length for values of $z$ between 1 and 2 inclusive and vanishes outside $W \cap C$, i.e. between $a$ and $a^{\prime}$ and $b^{\prime}$ and $b$. For convenience we assume $z=1 / 2$ and 5/2 at $a^{\prime}$ and $b^{\prime}$ respectively. We may, as before introduce coordinates in this tubular neighborhood $U$ so that $(z, 0,0, \ldots, 0), 0 \leqq z \leqq 3$, are the points on $C$. The last $2 m$ coordinates will be denoted by $x_{1}, \ldots, x_{2 m}$; they take on all values such that $r=\sum_{j=1}^{2 m} x_{j}^{2}<1$. Thus as the distance $d$ (in the metric on $M$ ) of $y \in U$ from $C$ varies from 0 to $\varepsilon$ the distance $r$ of its image in the coordinate cylinder from the $z$-axis goes from 0 to 1 ; that is, we suppose that $d=\left(\sum x_{j}^{2}\right)=\varepsilon r$. Use $\omega$ to denote the contact form both on $U$ and on the cylinder in the coordinate space $R^{2 m+1}$, then $\omega\left(Y_{z}\right)=0$ on $C$ since 
$C$ is an integral curve. We extend $Y_{z}$ to all the cylinder so as to be independent of $x_{1}, \ldots, x_{2 m}$; off the $z$-axis $\omega\left(Y_{z}\right) \neq 0$ in general.

Given the form $\omega$ on $U$ it will determine a vector field $E$ by the conditions that $i(E) d \omega=0$ and $\omega(E)=1$. Using this vector we may establish a 1-1 correspondence between $C^{\infty}$ functions on $M$ or $U$ and vector fields generating 1-parameter groups of automorphisms of the structure as follows: given a function $f$, let $\lambda(f)$ be the vector field determined by the conditions $i(\lambda(f)) d \omega=(i(E) d f) \omega-d f$ and $i(\lambda(f)) \omega$ $=0$. Then the vector field $\beta(f)=f E+\lambda(f)$ generates such an automorphism group and conversely. This characterization is due to Libermann [4] and will be used in the proof. Its validity results from the following equations based on the expression for the Lie derivative used above:

$$
\begin{aligned}
L_{\beta(f)} \omega & =i[\beta(f)] d \omega+d i[\beta(f)] \omega \\
& =f i(E) d \omega+i(E) d f \omega-d f+d f+d[i(\lambda(f))] \omega=(E f) \omega,
\end{aligned}
$$

that is, $\omega$ is invariant to within a scalar multiple. We now define a $C^{\infty}$ function $\alpha(z), 0 \leqq z \leqq 3$, with $\alpha(z) \geqq 0$, for all $z, \alpha(z) \equiv 1$ for $1 \leqq z \leqq 2$, and $\alpha(z)=0$ for every $z$ such that $Y_{z}=0$. We define a second $C^{\infty}$ function $\rho(r), 0 \leqq r \leqq 1$, which is 1 for $0 \leqq r \leqq 1 / 3$ and 0 for $r<1 / 2$ and monotone decreasing between these values. The form $\alpha \omega-i\left(Y_{z}\right) d \omega$ vanishes along the $z$-axis, i.e., when $x_{1}=\cdots=x_{2 m}=0$, on vectors tangent to the $z$-axis and thus in local coordinates we must have this form given by $\sum_{j=1}^{2 m} h_{j}(z) d x_{j}$ at points of the $z$-axis. When $0 \leqq z \leqq 1 / 2$ or $5 / 2 \leqq z \leqq 3$ the functions $h_{j}(z)$ thus defined vanish since both $\alpha$ and $Y_{z}$ vanish. Now let us define a function $f$ on our cylinder by

$$
f(z ; x)=\sum_{j=1}^{2 m} x_{j} h_{j}(z) \rho(r), \quad r=\sum_{j=1}^{2 m} x_{j}^{2} .
$$

Then $f$ is differentiable on the cylinder and in fact determines on $U$ a function which vanishes outside $W$ and thus can be extended to a $C^{\infty}$ function, also denoted $f$, on all of $M$ vanishing except inside $W . \beta(f)$ generates a 1-parameter group of automorphisms $\Phi_{t}$ of $M$ and we will show that it has the desired properties. This becomes clear if we show that along $C$, i.e. on the $z$-axis, $\beta(f)=Y_{z}$. To do this, first compute $d f$,

$$
d f=\sum_{j}\left\{h_{j}(z) \rho(r) d x_{j}+x_{j} \rho(r) \frac{d h_{j}}{d z} d z+x_{j} h_{j}(z) \rho^{\prime}(r) d r\right\} .
$$

However, for $r \leqq 1 / 3, \rho(r) \equiv 1$ so that $\rho^{\prime}=0$, thus on the $z$-axis $d f$ reduces to $d f$ $=\sum h_{j}(z) d x_{j}$, so that there $i\left(Y_{z}\right) d \omega=\alpha \omega-d f$. Moreover, we see that because $i(E) i\left(Y_{z}\right) d \omega=0, d f(E)=i(E) d f=\alpha \omega(E)=\alpha$ along this axis and thus $Y_{z}=\lambda(f)$ on $C$ according to our characterization of $\lambda(f)$ above. Since along the $z$-axis $f \equiv 0$, we must have $\beta(f)=\lambda(f)$; thus the equality of $\beta(f)$ and $Y_{z}$ along the curve $C$ is established. Hence the 1-parameter group of automorphisms $\Phi_{t}$ generated by $\beta(f)$ leaves 
all points outside $W$ fixed, $\Phi_{1}$ takes $p$ to $q$, and $\Phi_{t}, 0 \leqq t \leqq 1$, gives an isotopy to the identity. This completes the proof of the lemma.

It is easy to check that if two points $p$ and $q$ of $M$ are joined by a differentiably imbedded integral curve, then that curve may be extended slightly to a similar curve on which they are interior points. If they are joined by a piecewise integral curve on which each segment is an arc of the above type, then by using the same argument as in the transitivity of isotopies, which in fact implies it, we can establish the existence of an automorphism of $M$ taking $p$ to $q$ and isotopic to the identity, the isotopy leaving fixed all of $M$ except a compact set composed of the unions of the compact sets $\bar{W} \subset U$ (tubular neighborhood) of the type above for each of the segments. It is known that any two points of a manifold $M$ with a contact form $\omega$ can be joined by an integral curve of the desired type. This is essentially a theorem of Carathéodory; see Chow [2] or Boothby [6]. Then proceeding exactly as in the preceding section we may prove

THEOREM B. If $M$ is a manifold with a contact form and $p_{1}, \ldots, p_{k}$ and $q_{1}, \ldots, q_{k}$ are two sets of distinct points, then there is a map $\Phi$, which is an automorphism of the structure and which takes $p_{j}$ to $q_{j}$ for $j=1, \ldots, k . \Phi_{1}$ is isotopic to the identity $\Phi_{0}$ by an isotopy $\Phi_{t}, 0 \leqq t \leqq 1$, which consists of automorphisms and which leaves all of $M$ fixed except for a compact set of arbitrarily small volume.

\section{BIBLIOGRAPHY}

1. S. S. Chern, Pseudo-groupes continus infinis, Géométrie Différentielle: Colloque Internationale du C.N.R.S., Strasbourg, 1953.

2. W. L. Chow, Über Systeme von linearen partiellen Differentialgleichungen erster Ordnung, Math. Ann. 117 (1940), 98-105.

3. S. Kobayashi and K. Nomizu, Foundations of differential geometry, Interscience, New York, 1963.

4. P. Libermann, Sur les automorphismes infinitésimaux des structures symplectiques et des structures de contact, Colloque de Géométrie Différentielle Globale, Bruxelles, 1958.

5. J. Milnor, Topology from the differentiable viewpoint, The Univ. Press of Virginia, Charlottesville, 1965.

6. W. M. Boothby, On the integral curves of a linear differential form of maximum rank, Math. Ann. 177 (1968), 101-104.

7. Y. Hatakeyama, Some notes on the group of automorphisms of contact and symplectic structures, Tôhoku Math. J. 18 (1966), 338-347.

WASHINGTON UNIVERSITY, ST. LouIs, MissourI 